\title{
Therapie des nicht kleinzelligen Lungenkarzinoms (NSCLC) im Stadium IV
}

\author{
Karl-Matthias Deppermann ${ }^{\mathrm{a}} \quad$ Rudolf M. Huber $^{\mathrm{b}} \quad$ Eckart Laack $^{\mathrm{c}}$ Martin Reck $^{\mathrm{d}}$ \\ Monika Serke ${ }^{e}$ Joachim von Pawel ${ }^{f}$ \\ ${ }^{a}$ Klinik für Pneumologie, Ruppiner Klinikum GmbH, Neuruppin, \\ ${ }^{b}$ Medizinische Klinik Innenstadt, Universität München, \\ ${ }^{\mathrm{c}}$ Medizinische Klinik II, Universitätsklinikum Hamburg-Eppendorf, Hamburg, \\ dZentrum für Pneumologie und Thoraxchirurgie, Krankenhaus Großhansdorf, \\ ${ }^{e}$ Klinik für Pneumologie II, Lungenklinik Heckeshorn, Helios Klinikum Emil von Behring, Berlin, \\ ${ }^{f}$ Schwerpunkt Onkologie, Asklepios-Fachkliniken München-Gauting, Gauting, Deutschland
}

\section{Schlüsselwörter}

Nicht kleinzelliges Lungenkarzinom - Pharmakologie · Erlotinib $\cdot$ Kombinationstherapie $\cdot$ Monochemotherapie

\section{Key Words}

NSCLC · Pharmacology · Erlotinib - Combination therapy · Single agent therapy

\section{Erstlinientherapie}

Seit der 1995 publizierten Meta-Analyse der NSCLC Cooperative Group gelten platinhaltige Regime bei Patienten mit inoperablem, lokal fortgeschrittenem oder metastasiertem NSCLC in der Erstlinientherapie als Standard [1]. Die platinbasierte Chemotherapie besitzt dieser Analyse zufolge einen 1-Jahres-Überlebensbenefit von absolut $10 \%$ gegenüber einer alleinigen Supportivtherapie. Gleichzeitig wird eine effektivere Symptomkontrolle erreicht, die sich in einer verbesserten Lebensqualität äußert.

Dieses Ergebnis schlug sich in den Empfehlungen der American Society of Clinical Oncology (ASCO) nieder, die sich 1997 bei NSCLC-Patienten im Stadium IV und gutem Performance-Status (PS) für die platinhaltige Chemotherapie aussprach [2]. Allerdings wird die Verlängerung des Überlebens um wenige Monate mit einer gewissen Toxizität erkauft. Als problematisch gilt vor allem die Neurotoxizität, während die Nephrotoxizität heute beherrschbar ist. Auch Übelkeit und Erbrechen lassen sich mittlerweile dank effektiver Antiemetika gut kontrollieren. Erschwert wird die Cisplatin-basierte Therapie jedoch durch die für den Patienten belastende Hydrierung.

\section{Platinfreie Regime auf dem Vormarsch}

Die therapeutischen Optionen beim NSCLC im Stadium IV wurden in den letzten 10 Jahren durch moderne Zytostatika der 3. Generation erweitert. Diese Entwicklung warf die Frage auf, inwieweit die nebenwirkungsreichen Cisplatin-Regime durch nichtplatinhaltige Kombinationen zu ersetzen seien. In einer randomisierten Phase-III-Studie verglichen Georgoulias et al. [3] an 413 nicht zytostatisch vorbehandelten Patienten ein Regime aus Cisplatin und dem Zytostatikum der 3. Generation, Vinorelbin (VC), mit der modernen Kombination aus Docetaxel und Gemcitabin (DG) [3]. Beim medianen Überleben (9,7 vs. 9,0 Monate), der 1-Jahres-Überlebensrate (40,8 vs. $34,3 \%$ ) und der Ansprechrate $(39,2$ vs. $30,0 \%)$ bestand ein tendenzieller, aber nicht signifikanter Vorteil zugunsten der Cisplatin-haltigen Kombination. Allerdings war die Hämatoxizität (Grad 3/4) im VC-Arm signifikant höher $(\mathrm{p}<0,05)$; auch Übelkeit und Erbrechen traten signifikant häufiger auf als mit dem DG-Regime $(p=0,003)$. Die Autoren kamen deshalb zu dem Schluss, dass nichtplatinhaltige Regime in der Erstlinientherapie des fortgeschrittenen NSCLC insbesondere bei Cisplatin-Unverträglichkeit durchaus ihren Platz haben.

Zum selben Ergebnis kam auch eine randomisierte Phase-III-Studie von Laack et al. [4], in der das moderne Regime Gemcitabin/ Vinorelbin (GV) mit der Dreierkombination Gemcitabin/Vinorelbin/Cisplatin (GVP) verglichen wurde. Erwartungsgemäß war die Ansprechrate unter platinhaltiger Therapie signifikant höher als im platinfreien GV-Arm (28,3 vs. $13 \%$; $p=0,004)$. Dies schlug sich jedoch nicht in einer signifikanten Verbesserung des medianen Gesamtüberlebens und der 1-Jahres-Überlebensrate nieder, die in beiden Studienarmen vergleichbar ausfielen (Abb. 1). Zudem war die platinhaltige Tripeltherapie mit einer signifikant stärkeren Hämatotoxizität und einem ebenfalls signifikant häufigeren Auftreten 


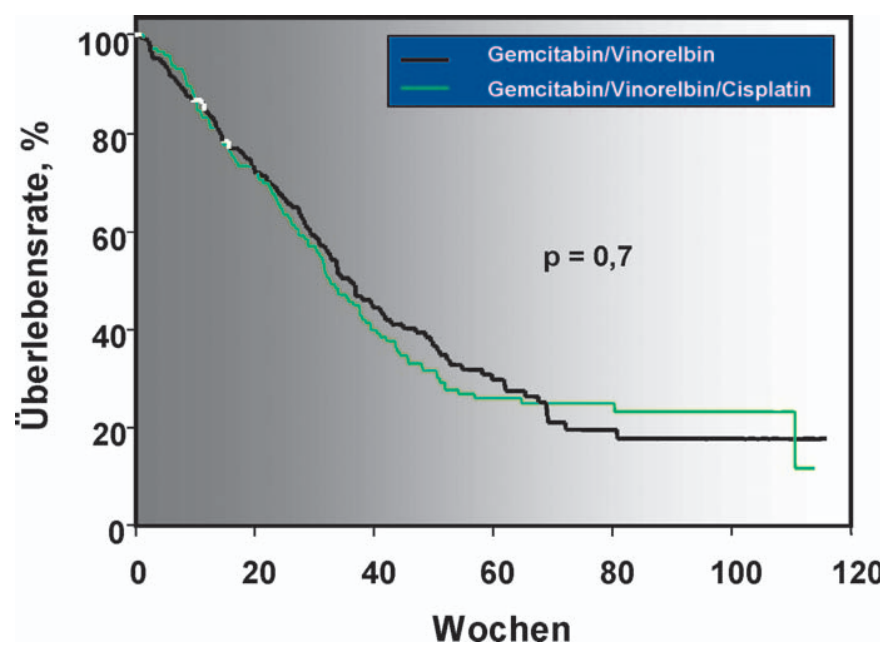

Abb. 1. Vergleichbare Überlebenszeiten unter einer Therapie mit Gemcitabin/Vinorelbin im Vergleich zur Dreierkombination mit Gemcitabin/Vinorelbin/Cisplatin (Laack).

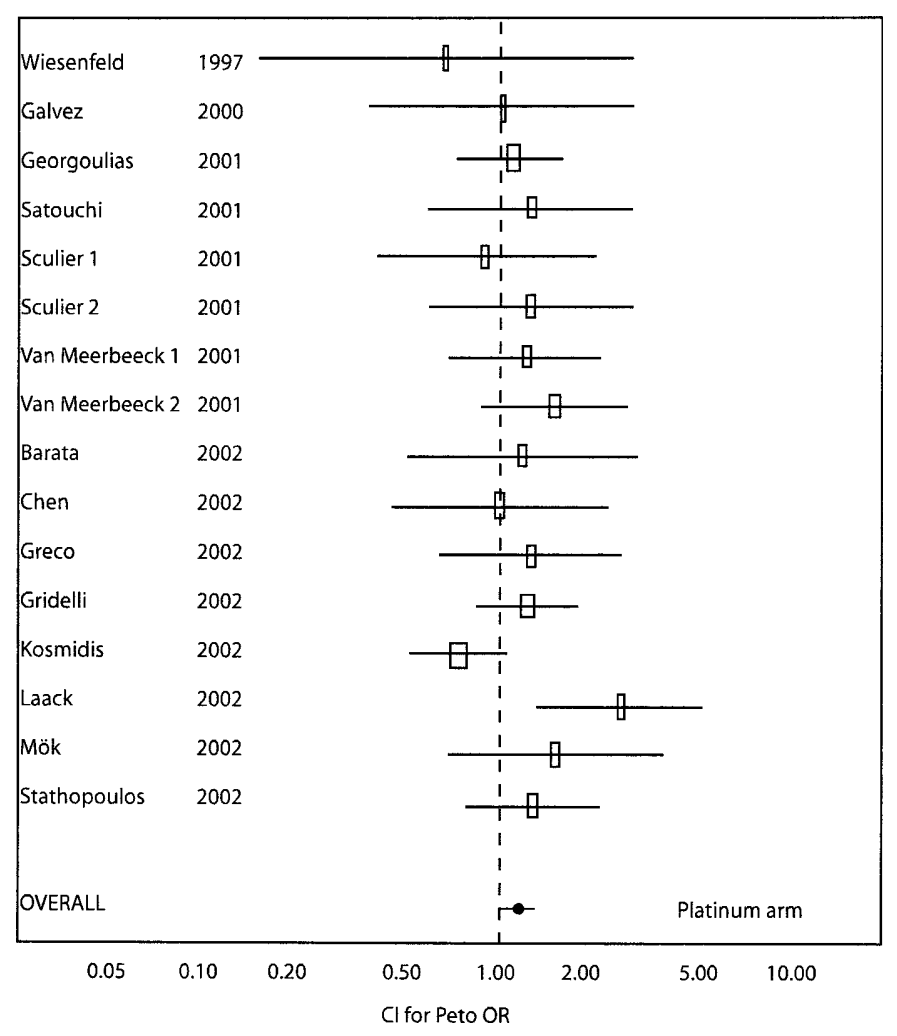

Abb. 2. Meta-Analyse zur Effektivität von platinhaltigen und platinfreien Therapieregimen [5]: signifikant höhere Ansprechraten mit der Cisplatin-basierten Therapie auch gegenüber modernen Kombinationen mit Zytostatika der 3. Generation (Serke).

von Alopezie, Übelkeit/Erbrechen und Nierenfunktionsstörungen assoziiert $(\mathrm{p}<0,0005)$. Somit zeigte diese Studie erneut, dass Dreierkombinationen in der Erstlinientherapie des NSCLC keinen Stellenwert haben, da sie verglichen mit Zytostatika-Doubletten keinen Überlebensvorteil bieten, aber mit einer höheren Toxizität behaftet sind.
Tab. 1. Meta-Analyse zur Effektivität von platinhaltigen und platinfreien Therapieregimen: vergleichbare Überlebensraten unter Cisplatin-basierter Therapie und modernen Kombinationen mit Drittgenerations-Zytostatika (Serke) [nach 5]

\begin{tabular}{ll}
\hline Verglichene Therapieregime & $\begin{array}{l}\text { 1-Jahres-Über- } \\
\text { lebensrate, } \%\end{array}$ \\
\hline Platinhaltige vs. nichtplatinhaltige Therapie & 34 vs. $29^{*}$ \\
Platinhaltige vs. nichtplatinhaltige Monotherapie & 35 vs. $25^{*}$ \\
$\begin{array}{l}\text { Platinhaltig vs. nichtplatinhaltige Mono- und Kombina- } \\
\text { tionstherapie (3. Generation) }\end{array}$ & $37 \%$ vs. $31^{*}$ \\
Platinhaltige vs. Zytostatika-Doubletten (3. Generation) & 36 vs. 35 \\
\hline
\end{tabular}

\section{Platinfreie Kombinationen - eine mögliche Alternative}

Auch eine aktuelle Meta-Analyse von 37 Studien mit über 7600 Patienten, in der platinhaltige und platinfreie Kombinationsregime auf ihre Effektivität und Verträglichkeit hin verglichen wurden, kam zu dem Schluss, dass platinhaltige Therapien signifikant häufiger Remissionen im Vergleich zu modernen platinfreien Regimen induzieren (Abb. 2) [5]. Bei der 1-Jahres-Überlebensrate dagegen waren die modernen platinfreien Zytostatika-Doubletten der platinhaltigen Therapie völlig ebenbürtig (Tab. 1).

Nachteilig waren die doppelt so hohe Anämie- und Granulozytopenierate und das dreimal so häufige Auftreten von Thrombozytopenien, Nephrotoxizität sowie Übelkeit und Erbrechen unter den platinhaltigen Regimen. Bei Nephrotoxizität, Neurotoxizität und febrilen Granulozytopenien schnitten platinbasierte Kombinationen und moderne platinfreie Doubletten vergleichbar ab.

Zwar ist diese Meta-Analyse mit Schwächen behaftet, da sie nicht anhand individueller Patientendaten, sondern nur auf Basis der publizierten Studienergebnisse erstellt wurde. Dennoch bewertete das ASCO-Komitee die Studienlage als eindeutig und kam in den aktualisierten Empfehlungen zu dem Schluss, dass bei Toxizitätsproblemen nichtplatinhaltige Regime in der Erstlinientherapie als Alternative zu platinbasierten Kombinationen betrachtet werden können [6]. Diese Empfehlung wurde seitdem durch neuere Studien nicht widerlegt. Doch sei daran erinnert, dass alle vorliegenden Studien auf den Nachweis der Überlegenheit platinhaltiger Kombinationen angelegt waren und nicht auf Nichtunterlegenheit oder Äquivalenz getestet wurden.

\section{Carboplatin oder Cisplatin?}

Mit Carboplatin steht seit rund 15 Jahren ein weiteres Platinderivat zur Verfügung, das beim NSCLC ebenfalls in einer Reihe von Studien geprüft wurde. Auch eine Meta-Analyse, für die 8 randomisierte Studien mit knapp 3000 Patienten ausgewertet wurden, widmete sich dem Vergleich von Cisplatin versus Carboplatin [7]. Der 5\%-ige Unterschied im Überleben zugunsten von Cisplatin war statistisch nicht signifikant $(\mathrm{p}=0,5)$, doch wurden mit Cisplatin-haltigen Kombinationen meist deutlich höhere Ansprechraten erreicht (Tab. 2). Steht das Erzielen einer Remission im Vordergrund, ist daher weiterhin Cisplatin-Doubletten der Vorzug zu ge- 
Tab. 2. Höhere Ansprechraten, aber vergleichbare Überlebensraten mit Cisplatin-Kombinationen im Vergleich zu Carboplatin-Regime (Serke) [nach 7]

\begin{tabular}{lllc}
\hline Referenz & Chemotherapie & Ansprechrate, \% & Mittlere Überlebenszeit, Monate \\
\hline Klastersky et al., 1990 [21] & PE/CE & $26 / 16$ & $7,0 / 6,3$ \\
Jelic et al., 2001 [22] & PMVd/CMVd & $37 / 36$ & $8,0 / 9,0$ \\
Rosell et al., 2002 [23] & PTax/CTax & $28 / 25$ & $9,8 / 8,2$ \\
Schiller et al., 2002 [24] & PTax/CTax & $21 / 17$ & $7,8 / 8,1$ \\
Zatloukal et al., 2003 [25] & PGem/CGem & $41 / 29$ & $8,8 / 8,0$ \\
Fossella et al., 2003 [26] & PDoce/CDoce & $32 / 24$ & $11,3 / 9,4$ \\
Mazzanti et al., 2003 [27] & PGem/CGem & $42 / 31$ & $10,4 / 10,8$ \\
Paccagnella et al., 2004 [28] & PMVb/CMVb & $43 / 39$ & $10,2 / 7,2$ \\
\hline
\end{tabular}

ben. Auch moderne Zytostatika der 3. Generation sollten mit Cisplatin kombiniert werden, da derartige Protokolle gegenüber dem entsprechenden Carboplatin-Regime mit einem signifikanten Überlebensvorteil von $11 \%$ assoziiert sind $(\mathrm{p}=0,039)$. Andererseits spielen beim individuellen Therapieentscheid Aspekte wie Verträglichkeit, Lebensqualität und Bequemlichkeit für den $\mathrm{Pa}$ tienten eine wesentliche Rolle und müssen daher bei der Abwägung zwischen Cisplatin und Carboplatin berücksichtigt werden.

\section{Therapie bei älteren Patienten bzw. bei Patienten} mit eingeschränktem Allgemeinzustand

\section{Kein therapeutischer Nihilismus bei älteren Patienten}

Das NSCLC tritt gehäuft im höheren Lebensalter auf: Statistiken zufolge liegt der Inzidenzgipfel bei beiden Geschlechtern zwischen dem 75. und dem 85. Lebensjahr und damit in einem Altersbereich, in dem vermehrt mit Komorbiditäten zu rechnen ist [8]. Altersbedingte Einschränkungen in verschiedenen Organsystemen wie Leber, Niere, Gefäßsystem, Nervensystem und Gastrointestinaltrakt führen im Allgemeinen zu einer schlechteren Verträglichkeit einer Chemotherapie. Das ASCO-Komitee plädiert in seinen aktuellen Leitlinien bei älteren Patienten (> 70 Jahre) und Patienten in eingeschränktem Allgemeinzustand für eine Monochemotherapie [6]. Die Effektivität eines solchen Ansatzes wurde in der Phase-IIIStudie ELVIS (Elderly Lung Cancer Vinorelbine Italian Study) belegt [9]: Die gut verträgliche Monotherapie mit Vinorelbin führte im Vergleich zur alleinigen Supportivbehandlung zu einer signifikanten Steigerung des medianen Überlebens (28 vs. 21 Wochen; $\mathrm{p}=0,03$ ) und der 1-Jahres-Überlebensrate (32 vs. $14 \% ; \mathrm{p}=0,03$ ) bei gleichzeitiger Verbesserung der Lebensqualität.

Eine Optimierung der mit der Monotherapie erreichten Ergebnisse wurde anschließend in MILES (Multicenter Italian Lung Cancer in the Elderly Study) versucht, einer großen randomisierten Phase-III-Studie, für die nahezu 700 mindestens 70-jährige Patienten randomisiert einer Monotherapie mit Gemcitabin oder Vinorelbin oder der Kombination beider Zytostatika zugeteilt wurden [10]. Über zwei Drittel der Teilnehmer besaßen mindestens 2 Begleiterkrankungen, knapp 20\% einen reduzierten PS. Die Studie ergab keinen Vorteil für die kombinierte Therapie (Tab. 3): Beide Monotherapien waren dem kombinierten Regime beim medianen und progressionsfreien Überleben und bei der 1-Jahres-Überlebensrate gleichwertig und wurden zudem erheblich besser vertra- gen. Die Rate toxizitätsbedingter Therapieabbrüche war allerdings in allen Armen relativ gering und mit je 7\% in den beiden Monotherapie-Armen kaum niedriger als bei kombinierter Therapie $(11 \%)$. Aufgrund der beiden italienischen Studien kann die früher im höheren Lebensalter kontrovers diskutierte Chemotherapie heute wegen der signifikanten Verbesserung von Überleben und Lebensqualität eindeutig als sinnvoll betrachtet werden. Therapie der Wahl für ältere Patienten mit Komorbiditäten ist eine Monotherapie mit einem modernen Zytostatikum.

\section{Kombinationstherapie auch im höheren Lebensalter?}

Neben dem Kollektiv der älteren Patienten mit Komorbiditäten gibt es auch die Gruppe der älteren Patienten in gutem Allgemeinzustand ohne wesentliche Begleiterkrankungen. Diese Patienten scheinen durchaus von einer Kombinationstherapie zu profitieren. In einer altersspezifischen Subanalyse der Studie 1594 der Eastern Cooperative Oncology Group (ECOG) zeigte sich, dass über 70-jährige Patienten in gutem Allgemeinzustand, die die strengen Einschlusskriterien der Studie erfüllten, in vergleichbarem Maße wie Jüngere von einer platinbasierten Therapie profitierten; relevante Unterschiede in progressionsfreiem und Gesamtüberleben sowie in der 1-Jahres-Überlebensrate zwischen beiden Altergruppen traten nicht zutage (Tab. 4). Auch die schwere therapieassoziierte Toxizität war bei den älteren Patienten kaum stärker ausgeprägt [11]. Zu gleich lautenden Ergebnissen kamen weitere retrospektive Analysen großer randomisierter Studien [12,13].

Entscheidend für die Indikationsstellung zu einer Kombinationstherapie ist vermutlich die Beschränkung auf gesunde ältere Patienten in gutem Allgemeinzustand, die von einer solchen Behandlung auch profitieren werden. Das Alter per se ist keine entscheidende Determinante. Wird eine platinhaltige Kombinationstherapie als indiziert betrachtet, ist Carboplatin vermutlich als günstigeres Platinderivat einzuschätzen.

Auch bei Patienten im PS 2, die rund 30\% aller Lungenkarzinompatienten stellen, kann die Chemotherapie aufgrund der Datenbasis der Meta-Analyse von 1995 als sinnvoll klassifiziert werden [1]. Allerdings ist, basierend auf einer Subgruppenanalyse, eine Cisplatin-haltige Kombination in normaler Dosierung für diese Gruppe nicht zu empfehlen [15].

Anders stellt sich die Situation für Carboplatin dar: So hatten Patienten im PS 2 in der Vergleichsstudie mit Paclitaxel vs. Paclitaxel/ Carboplatin deutliche Vorteile von der Kombination: Sowohl medianes Überleben (4,7 vs. 2,4 Monate) als auch 1-Jahres-Über- 
Tab. 3. MILES-Studie [10]: medianes Überleben, 1-Jahres-Überlebensrate, medianes progressionsfreies Überleben und Ansprechen (Reck)

\begin{tabular}{|c|c|c|c|c|c|}
\hline & \multirow[t]{2}{*}{ Vinorelbin } & \multirow[t]{2}{*}{ Gemcitabin } & \multirow{2}{*}{$\begin{array}{l}\text { Vinorelbin/ } \\
\text { Gemcitabin }\end{array}$} & \multicolumn{2}{|l|}{$\mathrm{p}$-Wert } \\
\hline & & & & $\begin{array}{l}\text { Vinorelbin vs. Vinorel- } \\
\text { bin/Gemcitabin }\end{array}$ & $\begin{array}{l}\text { Gemcibatin vs. Vinorel- } \\
\text { bin/Gemcitabin }\end{array}$ \\
\hline Mediane Überlebensdauer, Wochen & 36 & 28 & 30 & 0,93 & 0,69 \\
\hline 1-Jahres-Überlebensrate, \% & 38 & 28 & 30 & 0,93 & 0,69 \\
\hline Mediane progressionsfreie Überlebensdauer, Wochen & 18 & 17 & 19 & 0,32 & 0,31 \\
\hline Ansprechrate, \% & 18 & 16 & 21 & 0,47 & 0,18 \\
\hline
\end{tabular}

Tab. 4. Subgruppenanalyse der ECOG-Studie 1594 [11]: Ergebnisse in Abhängigkeit vom Lebensalter (Reck)

\begin{tabular}{llcc}
\hline & $<70$ Jahre & p-Wert \\
& (912 Patienten) & Jahre & (227 Patienten) \\
\hline Ansprechrate, \% & 22,1 & 24,5 & 0,76 \\
Mediane progressionsfreie Überlebensdauer, Monate (PS 0-1) & 3,71 & 3,75 & 0,37 \\
Mediane Überlebensdauer, Monate (PS 0-1) & 8,15 & 8,25 & 0,53 \\
1-Jahres-Überlebensrate, \% (PS 0-1) & 32,8 & 35,2 & 0,36 \\
6 Zyklen erhalten, \% der Patienten & 34 & 30 & 0,14 \\
Mediane Zyklenzahl & 4 & 3 & \\
\hline
\end{tabular}

lebensrate (18 vs. 10\%) ließen sich im Vergleich zur Paclitaxel-Monotherapie nahezu verdoppeln [16]. Platinfreie Kombinationen aus Zytostatika der 3. Generation sind dagegen zum jetzigen Zeitpunkt bei Patienten mit schlechtem PS nicht als günstig zu betrachten. Zusammenfassend kann eine Chemotherapie wegen der positiven Beeinflussung von tumorassoziierten Symptomen und Lebensqualität bei Patienten im PS 2 grundsätzlich als sinnvoll bewertet werden. Patienten in gutem Allgemeinzustand und ohne Begleiterkrankungen sollten mit Kombinationen aus Carboplatin und einem modernen Zytostatikum behandelt werden, während bei komorbiden Patienten eine Monotherapie mit Vinorelbin, Gemcitabin oder einem Taxan zu bevorzugen ist.

\section{Molekular gezielte Therapie mit neuen Substanzen bei älteren Patienten}

Mit den neuen «targeted drugs», die wesentlich verträglicher sind als die klassischen Zytostatika, steht mittlerweile eine weitere gut verträgliche Option für die Behandlung des NSCLC zur Verfügung. So waren $18 \%$ der in Großhansdorf im Rahmen des Extended Access Program (EAP) mit dem gegen die Tyrosinkinase des EGFR (epidermal growth factor receptor) gerichteten Erlotinib behandelten Patienten über 75 Jahre alt. Etliche von ihnen erhielten die Substanz als Erstlinientherapie. Die Erfahrungen sind positiv, da Erlotinib auch in diesem Kollektiv eine ausgeprägte Tumorreduktion und lang anhaltende Remissionen induzieren kann. Diese kasuistischen Daten werden mittlerweile durch Studienergebnisse belegt: Jackman et al. [17] erreichten mit einer Erlotinib-Monotherapie bei $60 \%$ der 68 evaluierbaren Patienten im Alter über 70 Jahre eine Tumorkontrolle (Response 12\%; Stabilisierung $48 \%$ ). Das mediane Überleben lag bei 10,3 Monaten.

Die positiven Ergebnisse sollen jetzt in der randomisierten TIE(Tarceva In Elderly)-Studie der Arbeitsgruppe Lungenkarzinom der AIO (Arbeitsgemeinschaft Internistische Onkologie) bei Patienten mit NSCLC IIIB/IV über 70 Jahre überprüft werden.

\section{Zweitlinientherapie}

\section{Mehr Optionen in der Zweitlinientherapie}

Erlotinib ist seit letztem Jahr zur Behandlung des lokal fortgeschrittenen oder metastasierten NSCLC nach Versagen mindestens einer Chemotherapie zugelassen. Das entspricht den Daten der Studie BR.21 des National Cancer Institute of Canada, in der Erlotinib bei 731 vorbehandelten Patienten in der Zweit- und Drittlinientherapie mit Plazebo verglichen wurde [18, 19]. Die Therapie mit dem EGFR-Inhibitor war der Plazebobehandlung bei allen Wirkparametern - von der Ansprech- und Stabilisierungsrate ( 9 vs. $<1 \%, p<0,001$ bzw. 35 vs. $27 \%$ ) bis hin zur Überlebenszeit (6,7 vs. 4,7 Monate; $\mathrm{p}<0,001)$ - überlegen.

Bislang gibt es noch keine klaren Empfehlungen, bei welchen Patienten in der zweiten Therapielinie eine Chemotherapie eingesetzt werden sollte und wann der EGFR-Inhibitor zu bevorzugen ist. In der BR.21-Studie war der Überlebensvorteil für Erlotinib dem einer Zweitlinienchemotherapie mit Docetaxel oder Pemetrexed zumindest vergleichbar. Es gibt jedoch Berichte, dass der EGFR-Inhibitor die Zytostatikaresistenz bei Patienten, die auf eine Chemotherapie nicht (mehr) ansprachen, durchbrechen kann. Inwieweit molekulare Marker zur Indikationsstellung einer EGFR-Inhibitor-Therapie herangezogen werden sollen, ist Gegenstand der Diskussion. Wie eine retrospektive Analyse der BR.21-Studie zeigte, sind für das Ansprechen relevante Faktoren weiterhin das weibliche Geschlecht, eine asiatische Herkunft und die Histologie eines Adenokarzinoms. Die höheren Ansprechraten spiegeln sich jedoch nicht in einem höheren Überleben wider. Die Risikoreduktion durch Erlotinib ist bei Männern und Frauen, Patienten mit Adeno- und Plattenepithelkarzinomen sowie bei Patienten unterschiedlicher Abstammung vergleichbar. Von den klinischen Parametern ist auch bezüglich des Überlebens ein «NieRaucher»-Status als günstig für den erfolgreichen Einsatz von EGFR-Inhibitoren anzusehen, jedoch haben auch Raucher von 
der Therapie profitiert. Es wird zusätzlicher klinischer Studien bedürfen, um herauszufinden, welche Patienten von welcher Therapiestrategie am ehesten profitieren.

\section{Neue Therapieansätze - Antiangiogenese als neues Therapieprinzip}

Ein interessanter neuer Therapieansatz ist der monoklonale Antikörper Bevacizumab, der alle 3 Isoformen des VEGF (vascular endothelial growth factor) bindet. VEGF, dem eine Schlüsselrolle bei der vom Tumor induzierten Neoangiogenese zukommt, wird von zahlreichen Tumoren überexprimiert und steigert so Tumorinvasivität und Metastasierung. In der ECOG-Studie 4599 wurde beim fortgeschrittenen NSCLC durch zusätzliche Gabe von Bevacizumab zu einer Standardtherapie mit Paclitaxel/Carboplatin eine signifikante Überlebensverlängerung im Vergleich zur alleinigen Chemotherapie erzielt (12,5 vs. 10,2 Monate; $p=0,007)$ [20]. In den USA avancierte die Tripeltherapie daraufhin zum Referenzarm der ECOG in neuen Studien. In Europa werden die Ergebnisse der ECOG-Studie derzeit in einer weiteren Phase-III-Studie überprüft, in der Bevacizumab mit Gemcitabin/Cisplatin kombiniert wird.

\section{Literatur}

1 Non Small Lung Cancer Cooperative Group: Chemotherapy in non-small lung cancer: a metaanalysis using updated data on individual patients from 52 randomized clinical trials. BMJ 1995;311: 899-909.

2 Clinical Practice Guidelines for the Treatment of Unresectable Non-Small Lung Cancer: Adopted on May 16, 1997 by the Amercian Society of Clinical Oncology. J Clin Oncol 1997;15:2996-3018.

3 Georgoulias V, et al: Vinorelbine plus cisplatin versus docetaxel plus gemcitabine in advanced non-small-cell lung cancer: a phase III randomized trial. J Clin Oncol 2005;23:2937-2945.

4 Laack E, et al: Randomized phase III study of gemcitabine and vinorelbine versus gemcitabine, vinorelbine and cisplatin in the treatment of advanced non-small-cell lung cancer: from the German and Swiss Lung Cancer Study Group. J Clin Oncol 2004;22:2348-2356.

5 D'Addaio $G$, et al: Platinum-based versus nonplatinum-based chemotherapy in advanced nonsmall-cell lung cancer: a meta-analysis of the published literature. J Clin Oncol 2005;23:18822883.

6 Pfister DG, et al: American Society of Clinical Oncology treatment of unresectable non-smallcell lung cancer guidelines: update 2003. J Clin Oncol 2004;22:5018-5020.

7 Hotta K, et al: Meta-analysis of randomized clinical trials comparing cisplatin to carboplatin in patients with advanced non-small cell lung cancer. J Clin Oncol 2004;22:3852-3859.

8 Arbeitsgemeinschaft Bevölkerungsbezogener Krebsregister in Deutschland: Krebs in Deutschland, 4. überarbeitete und aktualisierte Ausgabe Saarbrücken, 2004.

9 The Elderly Lung Cancer Vinorelbine Italian Study Group: Effects of vinorelbine on quality of life and survival of elderly patients with advanced non small cell lung cancer. J Natl Cancer Inst 1999;91:66-72.

10 Gridelli C, et al: Chemotherapy for elderly patients with advanced non-small-cell lung cancer: the Multicenter Italian Lung Cancer in the Elderly Study (MILES) phase III randomized study. J Natl Cancer Inst 2003;95:341-343.
11 Langer CJ, et al: Age-specific subanalyses of ECOG 1594: Fit elderly patients (70-80 years) with NSCLC do as well as younger pts $(<70)$. Proc ASCO 2003;22:abstr 2571.

12 Kelly K, et al: Should older patients receive combination chemotherapy for advanced stage nonsmall cell lung cancer? An analysis of Southwest Oncology Group trial 9509 and 9308. Proc ASCO 2001;20: abstr. 1313.

13 Hensing TA, et al: The impact of age on toxicity, response rate, quality of life, and survival in patients with advanced, stage IIIB or IV non small cell lung carcinoma treated with carboplatin and paclitaxel. Cancer 2003;98:779-788.

14 Koeller JM, et al: The impact of age, performance status (PS) and chemotherapy treatment on mortality in 1244 community-based advanced non-small cell lung cancer (NSCLC) patients. Proc ASCO 2003;22:abstr 2835.

15 Soria JC, et al: Do all patients with advanced non-small-cell lung cancer benefit from cisplatinbased combination therapy? Ann Oncol 2001;12: 1667-1670.

16 Lilienbaum R, et al: Single-agent (SA) versus combination chemotherapy (CC) in advanced non-small cell lung cancer (NSCLC): a CALGB randomized trial of efficacy qualitiy of life (QOL) and cost effectiveness. Proc ASCO 2002; 21:abstr 2.

17 Jackman D, et al: Phase II study of the EGFR tyrosine kinase inhibitor erlotinib in patients $>70$ years of age with previously untreated advanced non-small-cell lung carcinoma. Lung Cancer 2005;49(suppl 2):62(abstr O-188).

18 Shepherd FA, et al: A randomized placebo-controlled trial of erlotinib in patients with advanced non-small cell lung cancer (NSCLC) following failure on 1st line or 2 nd line chemotherapy. A National Cancer Institute of Canada Clinical Trials Group (NCIC CTG) trial. N Engl J Med 2005 14;135:123-132.

19 Tsao MS, et al: Erlotinib in lung cancer - molecular and clinical predictors of outcome $\mathrm{N}$ Engl J Med 2005;353:133-144.

20 Sandler AB, et al: Randomized phase II/III trial of paclitaxel (P) plus carboplatin (C) with or without bevacizumab (NSC704865) in patients with advanced non-sqameous non-small cell lung cancer (NSCLC): An Eastern Cooperative Oncology Group (ECOG) trial - E4599. Proc ASCO 2005;23:abstr LBA4.

21 Klastersky J, et al: A randomized study comparing cisplatin or carboplatin with etoposide in patients with advanced non-small-cell lung cancer: European Organization for Research and Treatment of Cancer Protocol 07861. J Clin Oncol 1990;8:1556-1562.

22 Jelic S, et al: Survival advantage for carboplatin substituting cisplatin in combination with vindesine and mitomycin $\mathrm{C}$ for stage IIIB and IV squamous-cell bronchogenic carcinoma: A randomized phase III study. Lung Cancer 2001;34:1-13.

23 Rosell R, et al: Phase III randomised trial comparing paclitaxel/carboplatin with paclitaxel/cisplatin in patients with advanced non-small-cell lung cancer: A cooperative multinational trial. Ann Oncol 2002;13:1539-1549.

24 Schiller JH, et al: Comparison of four chemotherapy regimens for advanced non-small-cell lung cancer. N Engl J Med 2002;346:92-98.

25 Zatloukal P, et al: Gemcitabine plus cisplatin vs gemcitabine plus carboplatin in stage IIIb and IV non-small cell lung cancer: A phase III randomized trial. Lung Cancer 2003;41:321-331.

26 Fossella F, et al: Randomized, multinational, phase III study of docetaxel plus platinum combination versus vinorelbine plus cisplatin for advanced non-small-cell lung cancer: The TAX326 Study Group. J Clin Oncol 2003;21:3016-3024.

27 Mazzanti P, et al: Randomized, multicenter, phase II study of gemcitabine plus cisplatin versus gemcitabine plus carboplatin in patients with advanced non-small cell lung cancer. Lung Cancer 2003;41:81-89.

28 Paccagnella A, et al: Cisplatin versus carboplatin in combination with mitomycin and vinblastine in advanced non small cell lung cancer. A multicenter, randomized phase III trial. Lung Cancer 2004;43:83-91. 